\title{
A COMPUTATIONAL METHOD FOR COMBINED OPTIMAL PARAMETER SELECTION AND OPTIMAL CONTROL PROBLEMS WITH GENERAL CONSTRAINTS
}

\author{
K. L. TEO ${ }^{1}$ AND C. J. GOH ${ }^{2}$
}

(Received July 8, 1987)

\begin{abstract}
In this paper, we consider a class of combined optimal parameter selection and optimal crentrol problems with general constraints. The first aim is to provide a unified approach to the numerical solution of this general class of optimisation problems by using the control parametrisation technique. This approach is supported by some convergence results. The second aim is to show that several different classes of optimal control problems can all be transformed into special cases of the problem considered in this paper. For illustration, four numerical examples are presented.
\end{abstract}

\section{Introduction}

Reference [6] presents a unified and efficient computational scheme for solving a general class of fixed terminal time optimal control problems involving joint continuous constraints on the state and control variables, and the terminal and interior point constraints on the state variable. The constraints are allowed to be of equality as well as inequality type, while the computational scheme is based on the control parametrisation technique (cf. [17], [18] and [19] for example). However, there are many practical problems which do not belong to this general class of optimal control problems. Examples include:

(i) optimal parameter selection problems;

\footnotetext{
${ }^{1}$ Department of Mathematics, University of Western Australia, Nedlands, Australia.

${ }^{2}$ Department of Industrial \& Systems Engineering, National University of Singapore, Kent Ridge, Singapore 0511, Republic of Singapore.

(C) Copyright Australian Mathematical Society 1989, Serial-fee code 0334-2700/89
} 
(ii) free terminal time optimal control problems, including minimum time problems;

(iii) minimax optimal control problems (problems with Chebyschev performance index);

(iv) boundary value control problems, including problems with periodic boundary conditions; and

(v) general combined optimal parameter and optimal control problems.

The aim of this paper is to extend the results of [6] to a more general class of optimisation problems so that all these important problems mentioned above can be regarded as special cases. Furthermore, as in [6], the algorithm presented in this paper also possesses the following important properties:

(i) the method always produces a suboptimal solution;

(ii) it is a unified approach, straightforward to apply and easily understood;

(iii) it is easily programmable and makes use of existing efficient optimisation algorithms;

(iv) the method has associated convergence results; and

(v) a software program has been developed for actual implementation of this algorithm [7].

For illustration, several examples are solved using the proposed computational scheme. We refer the interested reader to [5] for a list of relevant papers in computational methods (e.g. [4], [8], [11], [12], [13], [14] and [15]) for optimal control, and to [2], [3] and [5] for theoretical aspects of optimal control.

To close this section, we wish to note that from our computational experience, it was found that the computational scheme proposed in this paper does not appear to work well for free time optimal control problems if the optimal control is of the bang-bang type. For such problems, we propose to use the algorithm of [6] to solve a sequence of fixed terminal time optimal controls, following the idea of [19].

\section{Problem statement}

Consider a process described by the following differential equation defined on the fixed time interval $(0, T]$ :

$$
\dot{x}(t)=f(t, x(t), u(t), \varsigma),
$$

where $x \equiv\left[x_{1}, \ldots, x_{n}\right]^{\top} \in R^{n}, u \equiv\left[u_{1}, \ldots, u_{\tau}\right]^{\top} \in R^{r}, \varsigma \equiv\left[\varsigma_{1}, \ldots, \varsigma_{s}\right]^{\top} \in R^{s}$ are, respectively, the state, control, and organic parameters; $f \equiv\left[f_{1}, \ldots, f_{n}\right]^{\top} \in R^{n}$; and the superscript $T$ denotes the transpose.

The initial condition for the differential equation (1a) is

$$
x(0)=x^{0}(\varsigma)
$$


where $x^{0} \equiv\left[x_{1}^{0}, \ldots, x_{n}^{0}\right]^{\top}$ is a given function of the organic parameters $\zeta$.

Define

$$
Z \equiv\left\{\zeta \equiv\left[\varsigma_{1}, \ldots, \varsigma_{s}\right]^{\top} \in R^{s}: a_{i} \leq \varsigma_{i} \leq b_{\imath}, i=1, \ldots, s\right\}
$$

and

$$
U \equiv\left\{v \equiv\left[v_{1}, \ldots, v_{r}\right]^{\top} \in R^{r}: \alpha_{i} \leq v_{i} \leq \beta, i=1, \ldots, r\right\}
$$

where $a_{i}$ and $b_{i}, i=1, \ldots, s, \alpha_{i}$ and $\beta_{i}, i=1, \ldots, r$, are real numbers. Clearly, $Z$ and $U$ are compact and convex subsets of $R^{s}$ and $R^{r}$, respectively.

Any measurable function defined on $[0, T]$ with values in $U$ is called an admissible control. Let $\mathscr{U}$ be the class of all such admissible controls.

Let $L_{\infty}^{q}$ denote the Banach space $L_{\infty}\left([0, T], R^{q}\right)$ of all essentially bounded measurable functions from $[0, T]$ into $R^{q}$. Its norm is defined by

$$
\|\nu\|_{\infty} \equiv \operatorname{ess}_{t \in[0, T]}\left\{\sum_{i=1}^{q}\left(\nu_{i}(t)\right)^{2}\right\}^{1 / 2} .
$$

For each $(u, \varsigma) \in L_{\infty}^{r} \times R^{s}$, let $x(u, \varsigma)$ be an absolutely continuous function defined on $[0, T]$ which satisfies the differential equation (1a) almost everywhere on $(0, T]$ and the initial condition (1b). This function is called the solution of the system (1) corresponding to the combined control and parameter vector $(u, \varsigma) \in L_{\infty}^{r} \times R^{s}$.

We may now state our combined optimal parameter and optimal control problem as follows: given the system (1), find a combined control and organic parameter $(u, \zeta) \in \mathscr{U} \times Z$ such that the cost functional

$$
J(u, \varsigma) \equiv g_{0}(u, \varsigma)=\Phi_{0}(x(1), \varsigma)+\int_{0}^{1} \mathscr{L}_{0}(t, x(t), u(t), \varsigma) d t
$$

is minimised subject to equality constraints (in canonical form [6])

$$
g_{i}(u, \varsigma)=\Phi_{i}\left(x\left(\tau_{i}\right), \varsigma\right)+\int_{0}^{\tau_{i}} \mathscr{L}_{i}(t, x(t), u(t), \varsigma) d t=0, \quad i=1, \ldots, n_{e}
$$

and inequality constraints (in canonical form)

$$
g_{i}(u, \varsigma)=\Phi_{i}\left(x\left(\tau_{i}\right), \varsigma\right)+\int_{0}^{\tau_{i}} \mathscr{L}_{i}(t, x(t), u(t), \varsigma) d t \geq 0, \quad i=n_{e}+1, \ldots, N
$$

where $\Phi_{i}, i=0,1, \ldots, N$, and $\mathscr{L}_{i}, i=0,1, \ldots, N$, are given real valued functions; and $\tau_{i}$ is referred to as the characteristic time for the $i$ th constraint. For convenience, this optimisation problem is to be referred to as the problem $(P)$.

REMARK 2.1.

(i) (4a) and (4b) reduce to terminal equality constraints and terminal inequality constraints, respectively, if $\tau_{i}=T$ and $\mathscr{L}_{i}=0$. 
(ii) Similarly, the corresponding versions of (4a) and (4b) with $0<\tau_{i}<T$ and $\mathscr{L}_{i}=0$ are, respectively, equality interior point constraints and inequality interior point constraints.

(iii) The continuous equality constraint:

$$
h(t, x(t), u(t), \varsigma)=0, \quad t \in[0, T],
$$

is equivalent to (4a) with $\tau_{i}=T, \Phi_{i}(x(T), \varsigma)=0$ and

$$
\mathscr{L}_{i}(t, x(t), u(t), \varsigma)=(h(t, x(t), u(t), \varsigma))^{2} .
$$

(iv) Similarly, the continuous inequality constraint:

$$
h(t, x(t), u(t), \varsigma) \geq 0, \quad t \in[0, T],
$$

is equivalent to (4a) with $\tau_{2}=T, \Phi_{2}(x(T), \varsigma)=0$ and

$$
\mathscr{L}_{i}(t, x(t), u(t), \varsigma)=[\min \{h(t, x(t), u(t), \varsigma), 0\}]^{2} .
$$

Let $|\cdot|$ denote the usual norm in any finite dimensional Euclidean space. We assume throughout that the following conditions are satisfied:

(A1)

$$
\begin{array}{ll}
\Phi_{i}: R^{n} \times R^{s} \rightarrow R, & i=0,1, \ldots, N, \\
\mathscr{L}_{i}:[0, T] \times R^{n} \times R^{r} \times R^{s} \rightarrow R, & i=0,1, \ldots, N ;
\end{array}
$$

(A2) for each $i=1, \ldots, N$ and for each compact set $\Omega \times \Theta \subset R^{r} \times R^{s}$, there exists a positive constant $K$ such that $\left|\mathscr{L}_{i}(t, x, u, \varsigma)\right| \leq K(1+|x|)$ for all $(t, x, u, \zeta) \in[0, T] \times R^{n} \times \Omega \times \Theta$

(A3) $\mathscr{L}_{2}, i=0,1, \ldots, N$, are piecewise continuous on $[0, T]$ for each $(x, u, \varsigma) \in$ $R^{n} \times R^{r} \times R^{s}$, and continuously differentiable with respect to each of the components $x, u$ and $\varsigma$ for each $t \in[0, T]$;

(A4) $\Phi_{i}, i=0,1, \ldots, N$, are continuously differentiable with respect to each of the components $x$ and $\zeta$.

REMARK 2.2. From Theorem 1 of [9], we recall that system (1) admits a unique solution, $x(u, \zeta)$, corresponding to each $(u, \varsigma) \in L_{\infty}^{r} \times Z$, and hence for each $(u, s) \in \mathscr{U} \times Z$.

\section{Formulation of approximate problems}

In this section, we use the problem $(P)$ to construct a sequence of problems such that the solution of each of these approximate problems is a suboptimal solution to the problem $(P)$. This is done thorugh the discretisation of the control space. More precisely, we approximate each control by a zeroth order spline (i.e. a piecewise constant control) as follows. 
Consider a monotone non-decreasing sequence $\left\{S^{p}\right\}_{p=1}^{\infty}$ of finite subsets of $[0,1]$. For each $p$, let the $n_{p}+1$ points of $S^{p}$, denoted by $t_{0}^{p}, t_{1}^{p}, \ldots, t_{n_{p}}^{p}$, satisfy $t_{0}^{p}=0, t_{n_{p}}^{p}=1$, and $t_{k-1}^{p}<t_{k}^{p}, k=1,2, \ldots, n_{p}$. Furthermore, associated with each $S^{p}$ there is the obvious partition $I^{p}$ of $[0,1]$ defined by $I^{p} \equiv\left\{I_{k}^{p}: k=\right.$ $\left.1, \ldots, n_{p}\right\}$ where $I_{k}^{p}=\left[t_{k-1}^{p}, t_{k}^{p}\right)$. We choose $S^{p}$ such that $\lim _{p \rightarrow \infty} S^{p}$ is dense in $[0,1]$. This is equivalent to requiring that $\lim _{p \rightarrow \infty} \max _{k=1, \ldots, n_{p}}\left|I_{k}^{p}\right|=0$, where $\left|I_{k}^{p}\right|=t_{k}^{p}-t_{k-1}^{p}$, the length of the $k$ th interval.

Let $\mathscr{U}^{p}$ consist of all those elements from $\mathscr{U}$ which are piecewise constant and consistent with the partition $I^{p}$. It is clear that each $u \in \mathscr{U}^{p}$ can be written as:

$$
u(t)=\sum_{k=1}^{n_{p}} \sigma^{p, k} \chi_{I_{k}^{p}}(t), \quad t \in[0,1]
$$

where $\sigma^{p, k} \in U$ and $\chi_{I}$ denotes the characteristic function of $I$. This means that each control $u \in \mathscr{U}^{p}$ can be identified with $\sigma^{p}$, the $r n_{p}$ control parameter vector $\left[\sigma^{p, 1^{\top}}, \ldots, \sigma^{p, n} p^{\top}\right]^{\top}$ and vice versa. Thus, when no confusion can arise, we interchangeably refer to $u \in \mathscr{U}^{p}$ and $\sigma^{p} \in \mathscr{U}^{p}$.

Clearly, for each $u \in \mathscr{U}^{p}$, the control constraints defined in (3) are equivalent to

$$
\alpha_{i} \leq \sigma_{i}^{p, k} \leq \beta_{i}, \quad i=1, \ldots, r
$$

For brevity, define

$$
\theta^{p}=\left[\sigma^{p}, \varsigma\right]^{\top}
$$

Let $x\left(\theta^{p}\right)$ be the solution of the system (1) corresponding to the combined control and organic parameter vector $\theta^{p} \in \mathscr{U}^{p} \times Z$. Then, the constraints (4a) and $(4 \mathrm{~b})$ are reduced, respectively, to

$$
G_{i}\left(\theta^{p}\right)=\Phi_{i}\left(x\left(\theta^{p}\right)\left(\tau_{i}\right), \varsigma\right)+\int_{0}^{\tau_{1}} \widetilde{\mathscr{L}}_{2}\left(t, x\left(\theta^{p}\right)(t), \theta^{p}\right) d t=0, \quad i=1, \ldots, n_{e}
$$

and

$$
G_{i}\left(\theta^{p}\right)=\Phi_{i}\left(x\left(\theta^{p}\right)\left(\tau_{i}\right), \varsigma\right)+\int_{0}^{\tau_{i}} \widetilde{\mathscr{L}}_{i}\left(t, x\left(\theta^{p}\right)(t), \theta^{p}\right) d t \geq 0, \quad i=n_{e}+1, \ldots, N
$$

where $\widetilde{\mathscr{L}}_{i}, i=1, \ldots, N$, are obtained from $\mathscr{L}_{i}, i=1, \ldots, N$, in an obvious manner.

Let $\mathscr{F}^{p}$ be the set of all combined vectors $\theta^{p}=\left(\sigma^{p}, \varsigma\right)$ such that the constraints (8) are satisfied and that $\sigma^{p}$ are consistent with those elements in $\mathscr{U}^{p}$. Then, we can pose the approximate problem $\left(P_{p}\right)$ as follows: find a combined vector $\theta^{p} \in \mathscr{F}^{p}$ such that the cost functional

$$
\begin{aligned}
J\left(\theta^{p}\right) & \equiv G_{0}\left(\theta^{p}\right) \\
& =\Phi_{0}\left(x\left(\theta^{p}\right)(T), \varsigma\right)+\int_{0}^{T} \widetilde{\mathscr{L}}_{0}\left(t, x\left(\theta^{p}\right)(t), \theta^{p}\right) d t
\end{aligned}
$$


is minimised over $\mathscr{F}^{p}$, where $\widetilde{\mathscr{L}}_{0}$ is obtained from $\mathscr{L}_{0}$ in an obvious manner.

Note that for each $p$, the approximate problem $\left(P_{p}\right)$ can be viewed as a finite dimensional optimisation problem, namely, a nonlinearly constrained mathematical programming problem. This is the main theme behind the concept of the control parametrisation technique: to transform an optimal control problem to a mathematical programming problem. The discussion of the computational aspect will be given in Section 7 .

\section{Some convergence results}

In this section, we shall discuss convergence properties of the sequence of approximate optimal combined controls and organic parameter vectors. To be more precise, let $\left\{\theta^{p}\right\}_{p=1}^{\infty}$ be the sequence of optimal combined vectors to the sequence of finite dimensional optimisation problems $\left\{P_{p}\right\}_{p=1}^{\infty}$. Furthermore, let $\left\{\left(\bar{u}^{p}, \bar{\zeta}^{p}\right)\right\}_{p=1}^{\infty}$ be the corresponding sequence in $\mathscr{U} \times Z$. Clearly, each of them is a suboptimal solution to the original problem $(P)$, and is such that $J\left(\bar{u}^{p+1}, \bar{\zeta}^{p+1}\right) \leq J\left(\bar{u}^{p}, \bar{\zeta}^{p}\right)$ for all $p=1,2, \ldots$

To continue the discussion similar to that reported in Section 4 of [6], we need to introduce

DEFINITION 4.1. A combined vector $\theta^{p}$ is said to be $\varepsilon$-relaxed feasible if it satisfies the following $\varepsilon$-relaxed constraints:

$$
\begin{aligned}
-\varepsilon \leq G_{i}\left(\theta^{p}\right) & \leq \varepsilon, \quad i=1, \ldots, n_{e} \\
G_{i}\left(\theta^{p}\right) & \geq-\varepsilon, \quad i=n_{e}+1, \ldots, N .
\end{aligned}
$$

Let $\mathscr{F}_{\varepsilon}^{p}$ be the set which consists of all such $\varepsilon$-relaxed feasible combined vectors. Clearly, $\mathscr{F}^{p} \subset \mathscr{F}_{\varepsilon}^{p}$ for any $\varepsilon>0$.

We now consider the $\varepsilon$-relaxed version of the approximate problem $\left(P_{p}\right)$ as follows: find a combined vector $\theta_{\varepsilon}^{p} \in \mathscr{F}_{\varepsilon}^{p}$ such that the cost functional (9) is minimised over $\mathscr{F}_{\varepsilon}^{p}$.

For brevity, call this $\varepsilon$-relaxed problem the problem $\left(P_{p, \varepsilon}\right)$ and let $\theta_{\varepsilon}^{p, *}$ be an optimal combined vector of the problem $\left(P_{p, \varepsilon}\right)$. Since $\mathscr{F}^{p} \subset \mathscr{F}_{\varepsilon}^{p}$ for any $\varepsilon>0$,

$$
J\left(\theta_{\varepsilon}^{p, *}\right) \leq J\left(\theta^{p, *}\right)
$$

for any $\varepsilon>0$, where $\theta^{p, *}$ is an optimal solution to the problem $\left(P_{p}\right)$.

We may now specify the required additional condition mentioned earlier as follows.

(A5) For each positive integer $p$,

$$
\lim _{\varepsilon \downarrow 0} J\left(\theta_{\varepsilon}^{p, *}\right)=J\left(\theta^{p, *}\right) .
$$


This assumption is, indeed, not restrictive in view of the discussion following the assumption (A5) of [6].

For each $u \in \mathscr{U}$ and each $p$, define

$$
u^{p}(t)=\sum_{j=1}^{n_{p}} V(u)_{j}^{p} \chi_{I_{j}^{p}}(t), \quad t \in[0, T]
$$

where

$$
V(u)_{j}^{p} \equiv \frac{1}{\left|I_{j}^{p}\right|} \int_{I_{j}^{p}} u(s) d s, \quad j=1, \ldots, n_{p}
$$

and

$$
\left|I_{j}^{p}\right| \equiv\left|t_{j}^{p}-t_{j-1}^{p}\right|
$$

Since $U$ is compact and convex, it is clear that $V(u)^{p} \in U$. Hence, $u^{p} \in \mathscr{U}$.

We are now in a position to present the convergence results in the next two theorems. Their proofs are similar to those given for Theorem 4.1 and Theorem 4.2 of [6], respectively.

THEOREM 4.1. Let $\left(\bar{u}^{p}, \bar{\zeta}^{p}\right)$ be the optimal combined control and organic parameter vector of the approximate problem $\left(P_{p}\right)$. Suppose that there exists an optimal combined control and organic parameter vector, $\left(u^{*}, \varsigma^{*}\right)$, of the original problem $(P)$. Then

$$
\lim _{p \rightarrow \infty} J\left(\bar{u}^{p}, \bar{\varsigma}^{p}\right)=J\left(u^{*}, \varsigma^{*}\right) .
$$

THEOREM 4.2. Let $\left(\bar{u}^{p}, \bar{\zeta}^{p}\right)$ be the optimal combined control and organic parameter vector of the approximate problem $\left(P_{p}\right)$. Suppose that

$$
\lim _{p \rightarrow \infty}\left|\bar{u}^{p}(t)-u^{*}(t)\right|=0 \quad \text { almost everywhere on }[0, T]
$$

and

$$
\lim _{p \rightarrow \infty}\left|\vec{s}^{p}-s^{*}\right|=0
$$

Then, $\left(u^{*}, \varsigma^{*}\right)$ is an optimal combined control and organic parameter vector of the problem $(P)$.

\section{Model transformation}

In this section, our aim is to show that many different classes of optimal control problems can be transformed into special cases of problem $(P)$.

(i) Free terminal time problems (including minimum time problems):

$$
\min _{u(\cdot)} J=G_{0}=\Phi_{0}(x(T), T)+\int_{0}^{T} \mathscr{L}_{0}(t, x(t), u(t)) d t
$$


subject to the differential equation

$$
\frac{d x(t)}{d t}=f(t, x(t), u(t))
$$

with initial condition $x(0)=x^{0}$ and final condition $x(T)=x^{f}$.

If we treat $T$ as an unknown parameter and use the transformation $t=T \tau$, then (10) is converted to

$$
\min _{u(\cdot), T} J=G_{0}=\Phi_{0}(x(1), T)+\int_{0}^{1} T \mathscr{L}_{0}(\tau T, x(\tau), u(\tau)) d \tau
$$

subject to the differential equation

$$
\frac{d x(\tau)}{d \tau}=T f(\tau T, x(\tau), u(\tau))
$$

with initial condition $x(0)=x^{0}$ and final condition $x(1)=x^{f}$.

(ii) Minimax optimal control problems.

The state dynamical equations are as usual but the cost functional takes the form (Chebychev Performance Index):

$$
\min _{u(\cdot)} J=G_{0}=\max _{0 \leq t \leq 1} C(t, x(t), u(t))+\widehat{\Phi}_{0}(x(1))+\int_{0}^{1} \mathscr{L}_{0}(t, x(t), u(t)) d t .
$$

If we introduce an additional parameter

$$
S=\max _{0 \leq t \leq 1} C(t, x(t), u(t))
$$

then the minimax cost functional (14) is equivalent to

$$
\min _{u(\cdot), S} G_{0}=\Phi_{0}(x(1), S)+\int_{0}^{1} \mathscr{L}_{0}(t, x(t), u(t)) d t
$$

subject to

$$
S-C(t, x(t), u(t)) \geq 0, \quad t \in[0,1]
$$

where

$$
\Phi_{0}(x(1), S)=\widehat{\Phi}_{0}(x(1))+S .
$$

Here, the continuous constraint (17) can be further transformed into the canonical form:

$$
G_{n+1}=\int_{0}^{1}\{\min [S-C(t, x(t), u(t)), 0]\}^{2} d t=0 .
$$

(iii) Problems with periodic boundary conditions (and other interrelated boundary states).

The cost functional and the state dynamical equations take the usual form but the initial state and the final state are related through

$$
h(x(0), x(1))={ }^{\circ} 0 .
$$


In this case, if $n$ additional parameters are introduced:

$$
x(0)=x^{0}(\varsigma)=\varsigma,
$$

then, the constraint (19) is equivalent to

$$
h(\zeta, x(1))=0 .
$$

Once again, we have a special case of problem $(P)$.

\section{Smoothness of optimal control}

Since control parametrisation uses piecewise constant approximation, the control is in fact discontinuous. Often this suffices for many practical problems. However, continuous control, in fact control with any degree of smoothness, can be accomplished by MISER 2.0 (cf. [7]). This is, of course, done at the expense of more computational effort.

For example, if piecewise linear continuous control is desired, one only needs to introduce one extra set of differential equations: $\dot{u}(t)=v(t)$ with the initial conditions $u(0)=s_{s+1}$ where $s_{s+1}$ is an unknown organic parameter yet to be determined, $u$ is now a state function rather than a control function, and $v$ is the control function which are to be approximated by a piecewise constant function. In this way, the function $u$ is always piecewise linear.

Similarly, if we wish to approximate the optimal control by $C^{1}$ function we can introduce two extra sets of differential equations: $\dot{u}(t)=v(t), \dot{v}(t)=w(t)$ together with the initial conditions $u(0)=\varsigma_{s+1}, v(0)=\varsigma_{s+2}$, where $w(t)$ is now the piecewise constant control and $u(t)$ and $v(t)$ are now state variables. This process can continue, depending on how smooth our approximate control is desired to be.

\section{A unified computational approach}

The greatest virtue of solving constrained optimal control problems by control parametrisation is its generality and ease of implementation. In essence, after the parametrisation of control, the problem is reduced to that of a nonlinear mathematical programming problem. This, in turn, can be solved readily by some of the existing software such as $N L P Q L$ [16].

Like many other mathematical programming problems, this algorithm requires, at each iteration, the values of the cost functional and the constraints, and their respective gradients computed as follows.

Step 1. For a given $\theta^{p}$ integrate the state differential equation (1) forward in time from 0 to $\tau_{i}$. 
Step 2. Integrate the co-state differential equation

$$
\begin{gathered}
{\left[\dot{\lambda}^{i}\right]^{\top}=-\partial \mathscr{L}_{i} / \partial x-\left[\lambda^{i}\right]^{\top} \partial f / \partial x} \\
{\left[\lambda^{i}\left(\tau_{i}\right)\right]^{\top}=\frac{\partial \Phi_{i}\left(x\left(\theta^{p}\right)\left(\tau_{i}\right), \varsigma\right)}{\partial x\left(\theta^{p}\right)\left(\tau_{i}\right)}}
\end{gathered}
$$

backward in time from $\tau_{\imath}$ to 0 . Let the solution be denoted by $\lambda^{\imath}\left(\theta^{p}\right)$.

Step 3. The gradient is computed from

$$
\begin{aligned}
\nabla G_{i}\left(\theta^{p}\right)= & {\left[\lambda^{i}(0)\right]^{\mathfrak{T}} \frac{\partial x^{0}(\varsigma)}{\partial \theta}+\frac{\partial}{\partial \theta} \Phi_{i}\left(x\left(\theta^{p}\right)\left(\tau_{i}\right), \varsigma\right) } \\
& +\int_{0}^{\tau_{i}} \frac{\partial}{\partial \theta} H_{i}\left(t, x\left(\theta^{p}\right)(t), \theta^{p}, \lambda^{2}\left(\theta^{p}\right)(t)\right) d t
\end{aligned}
$$

where

$$
H_{i}\left(t, x, \theta, \lambda^{2}\right)=\mathscr{L}_{i}(t, x, \theta)+\left[\lambda^{i}\right]^{\top} f(t, x, \theta) .
$$

During actual computation, very often the control parametrisation is carried out on equal partitions of the interval $[0,1]$, i.e.

$$
u^{i}(t)=\sum_{j=1}^{n_{p}} \sigma_{i j} \chi_{\jmath}(t), \quad i=1, \ldots, r
$$

where $n_{p}$ is the number of equal subintervals, $\Delta=1 / n_{p}$ is the uniform partition length, and the characteristic function is given by

$$
\chi_{j}(t)= \begin{cases}1 & (j-1) \Delta \leq t \leq j \Delta \\ 0 & \text { otherwise }\end{cases}
$$

As such (23) can be written in a more specific form:

$$
\nabla_{\sigma_{i}}, G_{k}= \begin{cases}\int_{(j-\mathrm{r}) \Delta}^{j \Delta} \frac{\partial H_{k}}{\partial u_{i}} d t, & j<l \\ \int_{(j-1) \Delta}^{\tau_{i}} \frac{\partial H_{k}}{\partial u_{i}} d t, & j=l \\ 0, & j>l\end{cases}
$$

where $l=\operatorname{Int}\left(\tau_{i} / \Delta\right)$, and

$$
\nabla_{\zeta,} G_{k}=\left[\lambda^{k}(0)\right]^{\mathfrak{T}} \frac{\partial x^{0}(\varsigma)}{\partial \zeta_{j}}+\frac{\partial \Phi_{k}}{\partial \zeta_{j}}+\int_{0}^{\tau_{k}} \frac{\partial H_{k}}{\partial \zeta_{j}} d t .
$$

Based on these ideas, a general purpose FORTRAN program MISER 2.0 [7] has been developed to solve a general class of combined optimal parameter selection and optimal control problems discussed in the present paper.

\section{Illustration examples}

To illustrate the efficiency and versatility of the computational scheme proposed in this paper, four representative examples are solved using the software program MISER 2.0. 
EXAMPLE 1. Minimum time (brachistochrone problem with continuous state constraint):

$$
\begin{aligned}
& \min g_{0}=t_{f} \\
& \dot{x}(t)=(2 g y)^{1 / 2} \cos u, \quad x(0)=0, \\
& \dot{y}(t)=(2 g y)^{1 / 2} \sin u, \quad y(0)=0, \\
& \text { terminal state constraint: } x\left(t_{f}\right)=1, \\
& \text { continuous state constraint: } x \tan \phi+h \geq y, \quad \phi, h \text { constants. }
\end{aligned}
$$

Each analytical solution for this problem is available in [1]. The numerical results are obtained for the case of $n_{p}=20, \tan \phi=1 / 2$ and arbitrary initial guess by MISER 2.0. Their respective optimal costs are summarised as follows:

$\begin{array}{lllll} & & \text { Exact } t_{f}^{*} & & \text { Computed } t_{f}^{*} \\ \text { (i) Unconstrained, } & h=\infty & 0.5662 & & 0.5659 \\ \text { (ii) Constrained, } \quad h=0.1 & 0.5877 & & 0.5860 \\ \text { (iii) Constrained, } \quad h=0.0 & 0.6913 & & 0.6896\end{array}$

Note that there is some negligible constraint violation in each case, which contributes to the fact that the computed $t_{f}^{*}$ is slightly less than the exact $t_{f}^{*}$.

EXAMPLE 2. Minimax optimal control problem:

$$
\begin{aligned}
& \min _{u(\cdot)} g_{0}=\left[x_{2}^{2}(t)+u^{2}(t)\right] \\
& \dot{x}_{1}(t)=x_{2}(t) \quad x_{1}(0)=0, \\
& \dot{x}_{2}(t)=u(t) \quad x_{2}(0)=0, \\
& \text { terminal state constraint } x_{1}(1)=1, x_{2}(1)=0 .
\end{aligned}
$$

The exact analytical solution for this problem is available in [10]. The solution obtained from the present calculations using $n_{p}=10$ and arbitrary initial guess is $g_{0}^{*}=16.61$ as compared to the exact value of $g_{0_{\text {exact }}^{*}}^{*}=16.68$.

EXAMPLE 3. Free initial value and periodic boundary conditions optimal control.

This problem was previously considered by Dolezal [4] using quasilinearisation and a first order gradient algorithm.

$$
\begin{aligned}
& \min g_{0}=\frac{1}{2} \int_{0}^{1}\left(x_{1}^{2}+x_{2}^{2}+u^{2}\right) d t \\
& \dot{x}_{1}=x_{2} \quad x_{1}(0)=\text { free, } \\
& \dot{x}_{2}=-x_{1}+u+\left(1-x_{1}^{2}\right) x_{2} \quad x_{2}(0)=1.0 .
\end{aligned}
$$

We consider three cases:

$$
\begin{array}{ll}
\text { Case (i) } & x_{1}(0)=\text { free, no constraint on control } \\
\text { Case (ii) } & x_{1}(0)=\text { free, } \quad|u| \leq 0.5 \\
\text { Case (iii) } & \text { periodic boundary condition } x_{1}(0)-x_{1}(1)=0 .
\end{array}
$$


The commuted results using $n_{p}=20$ are compared with Dolezal's results as follows.

\begin{tabular}{lcccc} 
& $\begin{array}{c}\text { Dolezal } \\
g_{0}^{*}\end{array}$ & $\begin{array}{c}\text { Solution } \\
x_{1}^{*}(0)\end{array}$ & $\begin{array}{c}\text { Present } \\
g_{0}^{*}\end{array}$ & $\begin{array}{c}\text { Solution } \\
x_{1}^{*}(0)\end{array}$ \\
\hline & & & & \\
Case (i) & 0.6506 & 0.3792 & 0.6509 & 0.3814 \\
Case (ii) & 0.6694 & 0.4341 & 0.6695 & 0.4350 \\
Case (iii) & 1.019 & 0.9489 & 1.019 & 0.9493
\end{tabular}

EXAMPLE 4. A minimum time problem with singular arc.

The equations of motion of a vehicle with velocity of magnitude one for which the angular rate of the velocity is controlled are [5]:

$$
\dot{x}=\cos \phi, \quad \dot{y}=\sin \phi, \quad \dot{\phi}=u \text {. }
$$

Assume $|u| \leq 1$

Consider the problem of transferring the vehicle from initial conditions $x(0)=$ $4, y(0)=0, \phi(0)=\pi / 2$ to $x\left(t_{f}\right)=y\left(t_{f}\right)=0$ in minimum time $t_{f}$. This is a problem appearing on page 57 of $[5]$.

The exact minimum time and exact optimal control (Figure 1(i)) are given, respectively, by $t_{f}^{*}=4.7391$ and

$$
u^{*}(t)= \begin{cases}1, & 0 \leq t \leq t_{s}=1.9106 \\ 0, & t_{s}<t \leq t_{f}^{*}\end{cases}
$$

The optimal phase trajectory is plotted in Figure 1(iii).

With $n_{p}=20$ and arbitrary initial guess, MISER 2.0 is used to solve the problem. The computed minimum time is $t_{f}^{*}=4.7389$ and the computed optimal control and optimal phase trajectory are plotted in Figure 1(ii) and Figure 1(iv), respectively. Note that the computed state trajectory coincides almost identically with the analytical one. 


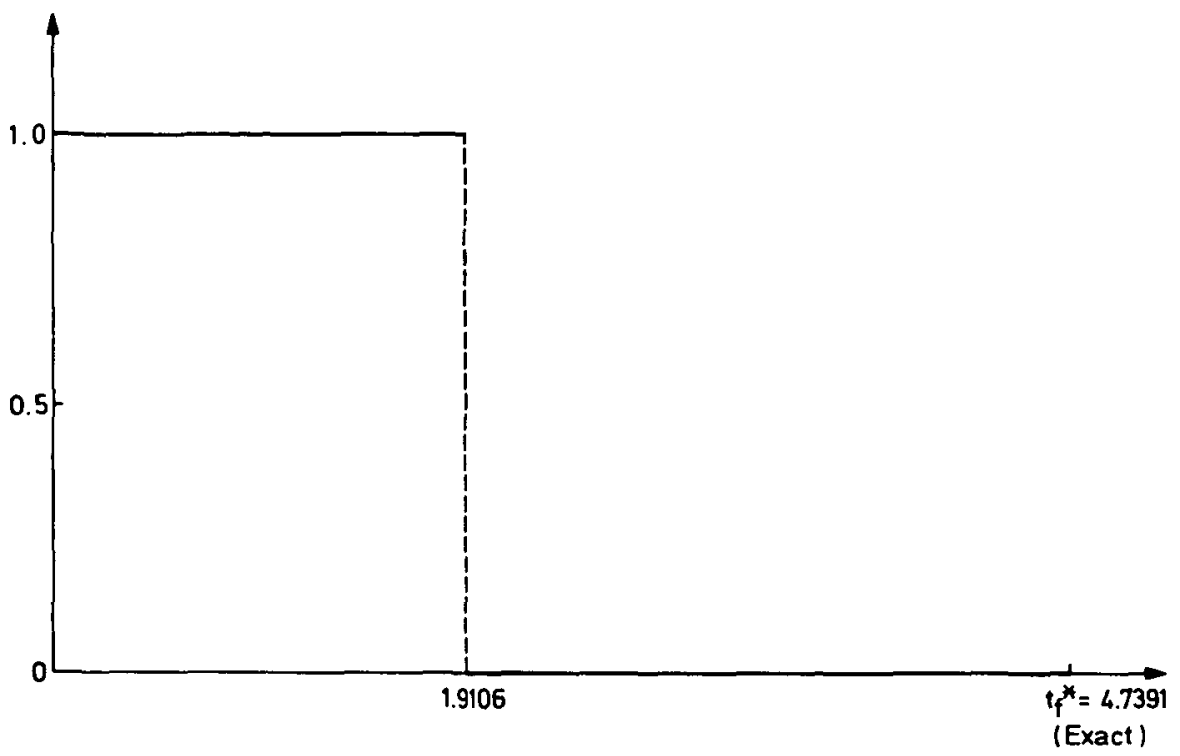

FIGURE 1(i). Exact optimal control.

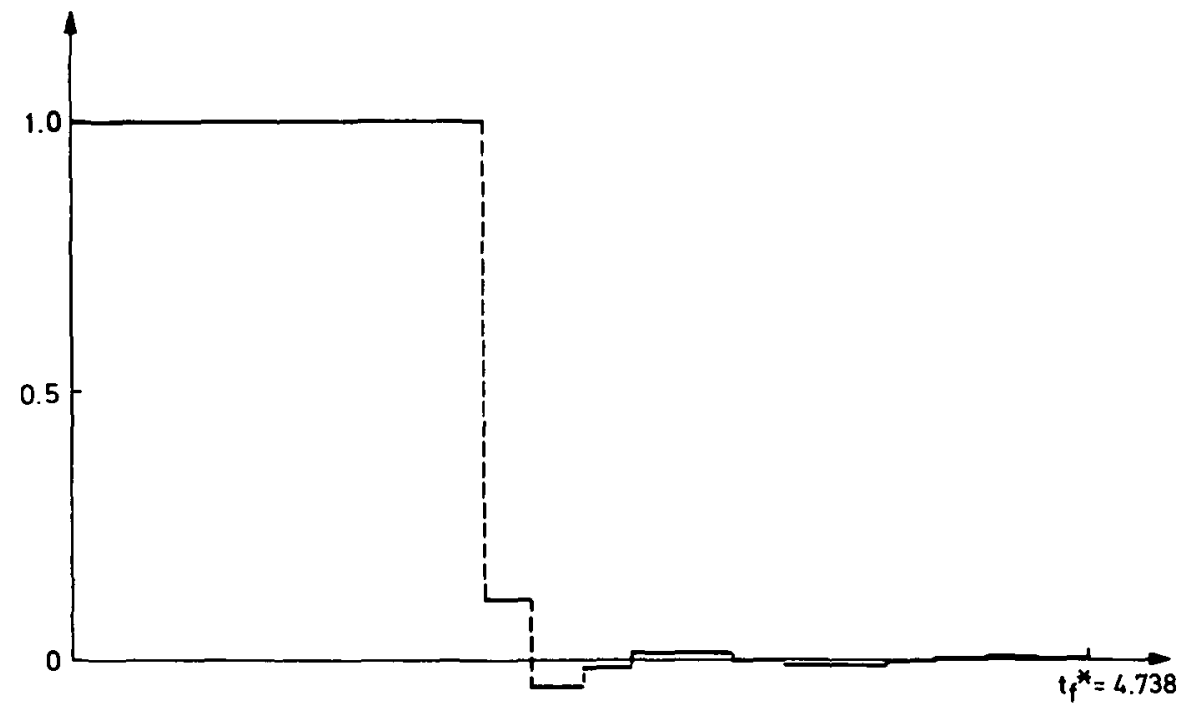

FIGURE 1(ii). Computed optimal control: $n_{p}=20$. 


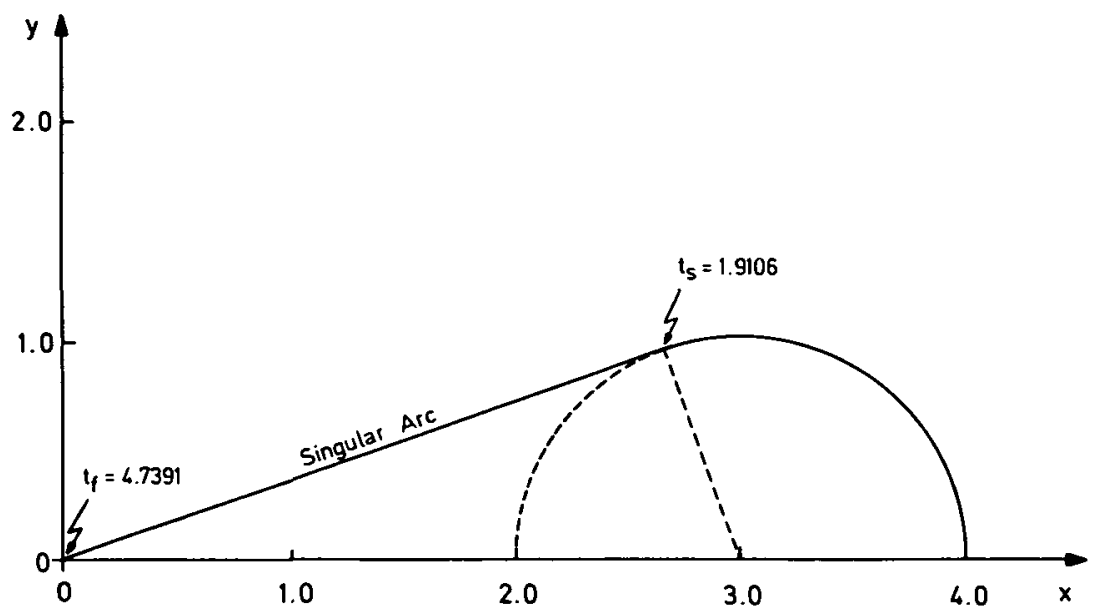

FIGURE 1(iii). Exact optimal phase trajectory.

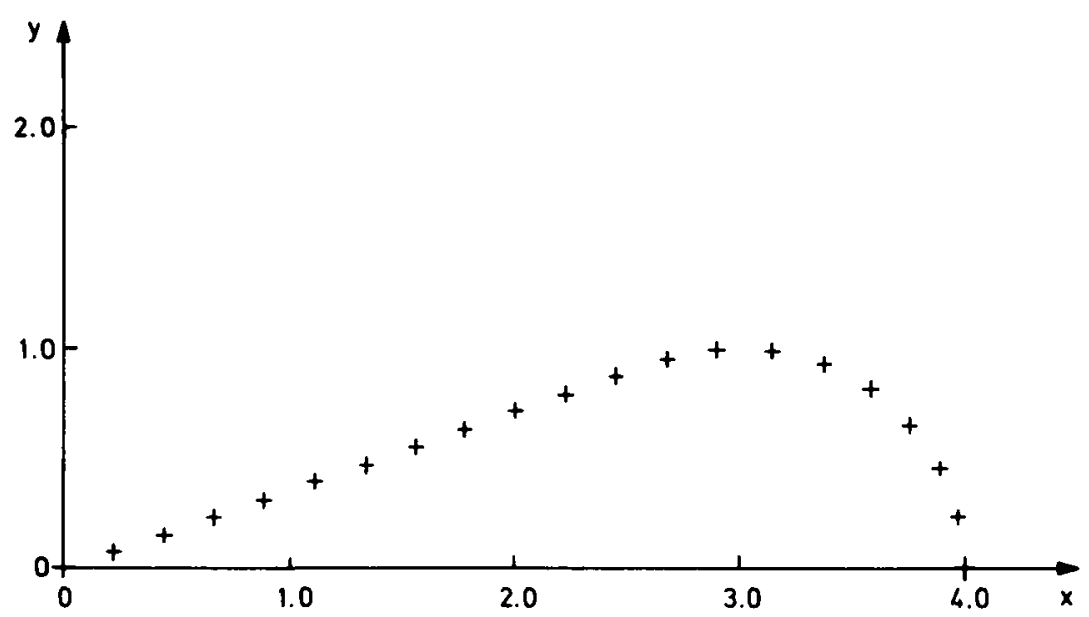

FIGURE 1(iv). Computed optimal phase trajectory: $n_{p}=20$.

\section{References}

[1] A. Bryson and Y. C. Ho, Applied Optimal Control (Halsted Press, New York, 1969).

[2] L. Cesari, Optimization-Theory and Applications (Springer-Verlag, New York, 1983).

[3] B. D. Craven, Mathematical Programming and Control Theory (Chapman and Hall, London, 1978). 
[4] J. Dolezal, "On the solution of optimal control problems involving parameters and general boundary conditions", Kybernetika 17 (1981), 71-81.

[5] W. H. Fleming and R. W. Rishel, Deterministic and Stochastic Optimal Control (SpringerVerlag, Berlin, 1975).

[6] C. J. Goh and K. L. Teo, "Control parametrization: A unified approach to optimal control problem with general constraints", Automatica 24 (1988), 3-18.

[7] C. J. Goh and K. L. Teo, MISER: An optimal control software (Applied Research Corporation, Nat. University of Singapore, Singapore, 1987).

[8] S. Gonzalez and A. Miele, "Sequential gradient-restoration algorithm for optimal control problems with general boundary conditions", J. Optim. Theory Appl. 26 (1978), 395-425.

[9] H. Halkin, "Mathematical foundations of system optimisation", in Topics in Optimisation (ed. G. Leitmann), (Academic Press, New York, 1967).

[10] K. Holmaker, "A minimax optimal control problem", J. Optim. Theory Appl. 28, No. 3 (1979), 391-410.

[11] A. Miele, "Gradient algorithms for the optimization of dynamic systems", Control and Dynamic Systems: Advances in Theory and Applications 16 (ed. C. T. Leondes), (Academic Press, New York, 1980), 1-52.

[12] A. Miele and T. Wang, "Primal-dual properties of sequential gradient-restoration algorithms for optimal control problems, Part 1, Basic problems", in Integral Methods in Sci. and Engng. (ed. F. R. Payne et al.), (Hemisphere Publishing Corporation, Washington, D.C., 1986), 577-607.

[13] A. Miele and T. Wang, "Primal-dual properties of sequential gradient-restoration algorithms for optimal control problems, Part 2, General problem", J. Math. Anal. Appl. 119 (1986), 21-54.

[14] N. Nedeljkovic, "The LQRE computational method in optimal control theory", Ph.D. Thesis, Murdoch University, 1985.

[15] E. Polak, Computational Methods in Optimization (Academic Press, New York, 1971).

[16] K. Schittkowski, "NLPQL: A FORTRAN subroutine solving constrained nonlinear programming problems", Oper. Res. Annals 5 (1985), 485-500.

[17] H. R. Sirisena, "Computation of optimal controls using a piecewise polynomial parametrization", IEEE Trans. Automat. Control AC-18 (1973), 409-411.

[18] H. R. Sirisena and F. S. Chou, "Computation of constrained optimal controls using parametrization techniques", IEEE Trans. Automat. Control AC-19 (1974), 431-433.

[19] K. L. Teo and C. C. Lim, "Time optimal control computation with application to ship steering", J. Optim. Theory Appl. 56 (1988), 145-156. 